\title{
ESPASMO HEMIFACIAL: RESULTADOS DO TRATAMENTO CIRÚRGICO EM 14 CASOS
}

\author{
Rui R. D. Carvalho * \\ Salvador V. Oliveira ** \\ JUAN R. C. RODRIGUES **
}

O espasmo hemifacial é uma hipercinesia paroxística limitada aos músculos da face inervados pelo $7 .^{\circ}$ nervo craniano. Caracteriza-se por contrações clônicas, às vezes adquirindo aspecto tônico semelhante à excitação farádica do nervo facial. Em geral, inicialmente afeta o músculo orbicular das pálpebras, porém, com o tempo, se difunde comprometendo todos os músculos de um só lado da face. Estas contrações independem da vontade do paciente, mas podem ser desencadeadas pelos movimentos faciais e exacerbadas sob tensão psíquica. Difere da sincinesia pós-paralítica porque a contratura persiste após cessado o movimento facial voluntário. Raramente é bilateral ${ }^{6}$ e não é acompanhada de paralisia ou paresia facial. Pode ser observada durante o sono ${ }^{7}$.

A fisiopatologia desta doença tem apresentado aspectos conflitantes. Wilson ${ }^{20}$ escreve: "comum como é, ambos causa e patogênese são obscuros, enquanto dados patológicos são raros e ambiguos." Wartenberg ${ }^{19}$ concluiu que tanto no espasmo hemifacial como na sincinesia pós-paralítica a lesão estaria ao nível do núcleo do facial. Esta opinião é corroborada por Greenwood ${ }^{9}$. Teasdal e Salman ${ }^{18}$ baseados em estudos eletromiográficos de 8 pacientes com sincinesia pós-paralítica concluiram por um maldirecionamento das fibras regeneradas, enquanto Magun e Esslen ${ }^{14}$, em estudos semelhantes de 15 pacientes de espasmo hemifacial (não paralítico), concluiram pela existência de estímulos transaxonais devidos a lesão da bainha isolante do axônio normal. Este curto-circuito far-se-ia entre as porções sensitivas e motora do facial sendo, portanto, localizado no seu segmento intracraniano.

Krause ${ }^{12}$, em 1912, observou tremores na face de pacientes com tumor do ângulo ponto-cerebelar. Cushing ${ }^{2}$, em 1917, relatou 4 casos de espasmo hemifacial em 30 neurinomas do acústico afirmando que "os sintomas irritativos referentes ao nervo facial têm sido mais freqüentemente observados do que é geralmente acreditado". Outros autors relatam malformações vasculares em contato com o nervo facial no ângulo ponto-cerebelar $1,3,5,7,11$, presença de doença de Paget ${ }^{6}$ e aracnoidite ${ }^{13}$. O espasmo hemifacial poderá ser acompanhado de nevralgias do trigêmeo ${ }^{6,10}$ ou do geniculado ${ }^{11}$.

Disciplina de Neurocirurgia da Faculdade de Ciências Médicas da Santa Casa de São Paulo: * Professor; ** Assistentes. 
A neurotomia seletiva de ramos do facial ${ }^{8,15,16}$ ou secção parcial do tronco do mesmo ${ }^{17}$, em seu segmento extracraniano, são acompanhadas de recidiva assim que a regeneração do nervo se estabelece. Na secção do facial no forame estilomastóideo e anastomose com o espinhal e hipoglosso a regeneração não é acompanhada de recidiva do espasmo ${ }^{4}$. Em ambos os casos a melhora do espasmo se faz a custa de paralisia. Tais fatos sugerem que o curto-circuito causador do espasmo esteja localizado nos segmentos intratemporal ou intra-craniano do nervo.

Gardner e Sava ${ }^{7}$ exploraram o nervo facial no ângulo ponto-cerebelar de 19 pacientes com espasmo hemifacial, tendo encontrado compressão do nervo em 14 casos: a simp'es neurolise produziu a cura em 12 pacientes e melhora satisfatória em mais 3 . Não houve melhora em 2 e outros 2 apresentaram recidiva.

Este trabalho tem o propósito de apresentar os achados cirúrgicos e resultados na exploração e neurolise do facial no ângulo ponto-cerebelar de 14 pacientes (Tabela 1) por nós observados.

\section{CASUISTICA E RESULTADOS}

Oito pacientes eram do sexo feminino e 6 do masculino, variando a idade entre 27 anos e 69 anos, com média de 46,4 anos. Os lados foram igualmente comprometidos. A duração do espasmo variou entre 1 ano e 8 anos, com média de 3,6 anos. Em todos foi realizada exploração do ângulo ponto-cerebelar por craniectomia occipital unilateral sob anestesia geral e em posição sentada. O primeiro paciente foi operado em 1963 e o último em setembro de 1972. Todos têm seguimento de pelo menos um ano, com excecão do último paciente operado.

\begin{tabular}{|c|c|c|c|c|c|c|c|c|c|}
\hline \multirow[t]{2}{*}{ Caso } & \multirow[t]{2}{*}{ Nome } & \multirow{2}{*}{$\begin{array}{l}\text { Idade } \\
\text { (anos) }\end{array}$} & \multirow[t]{2}{*}{ Sexo } & \multirow[t]{2}{*}{ Cor } & \multirow{2}{*}{\multicolumn{2}{|c|}{ Registro }} & \multicolumn{2}{|c|}{ Espasmo } & \multirow{2}{*}{$\begin{array}{l}\text { Data da } \\
\text { operação }\end{array}$} \\
\hline & & & & & & & lado & duração & \\
\hline 1 & SMR & 27 & $\mathbf{F}$ & B & SCN & 13060 & $\mathbf{E}$ & 3 & 29.10 .63 \\
\hline 2 & $\mathrm{ALC}$ & 28 & $\mathrm{M}$ & B & $\mathrm{SC}$ & 674705 & $\mathbf{E}$ & 1 & 05.04 .67 \\
\hline 3 & JAA & 49 & $F$ & B & $\mathrm{SC}$ & 365917 & $\mathbf{E}$ & 3 & 29.08 .68 \\
\hline 4 & WCF & 36 & $\mathbf{F}$ & B & $\mathrm{SC}$ & 730645 & $\mathbf{D}$ & 6 & 19.10 .68 \\
\hline 5 & SFH & 58 & $F$ & B & $\mathrm{SC}$ & 721085 & $\mathbf{E}$ & 3 & 18.03 .70 \\
\hline 6 & SLM & 27 & $F$ & $\mathrm{~B}$ & SC & 790960 & D & 3 & 08.06 .70 \\
\hline 7 & ICR & 49 & $\mathbf{F}$ & B & $\mathrm{SC}$ & 783104 & D & 8 & 05.10 .70 \\
\hline 8 & DSA & 68 & $\mathbf{M}$ & B & $\mathrm{HH}$ & 5578 & D & 1 & 31.03 .71 \\
\hline 9 & YNK & 40 & $\mathbf{F}^{1}$ & B & SC & 816112 & $\mathbf{E}$ & 5 & 05.04 .71 \\
\hline 10 & JLA & 54 & M & B & $\mathrm{HH}$ & 7590 & D & 5 & 23.04 .71 \\
\hline \pm 1 & JAC & 47 & M & B & $\mathrm{HH}$ & 7619 & $\mathbf{E}$ & 1 & 26.04 .71 \\
\hline 12 & APMR & 69 & $\mathbf{F}$ & B & SC & 837350 & D & 2 & 03.01 .72 \\
\hline 13 & PB & 58 & $\mathbf{M}$ & $\mathbf{B}$ & $\mathrm{HH}$ & 12054 & $\mathrm{D}$ & 5 & 17.01 .72 \\
\hline 14 & PBS & 44 & $\mathbf{M}$ & $\mathrm{B}$ & $\mathbf{H H}$ & 14510 & $\mathbf{E}$ & 4 & 27.09 .72 \\
\hline
\end{tabular}

Tabela 1 - Dados de identificação dos 14 pacientes com espasmo hemifacial. Registro: SC = Santa Casa de São Paulo; SCN = Santa Casa de São Paulo, Neurologia; $H H=$ Hospital Heliópolis, São Paulo. Espasmo: duração em anos. 
Em 6 pacientes (casos 1, 2, 4, 10, 13, 14) nada de anormal foi encontrado na fossa posterior; destes pacientes, um permaneceu inalterado (caso 1) e outro apresentou recidiva 10 meses mais tarde (caso 4); o paciente P.B.S. (caso 14) estava bem 4 meses após o ato cirúrgico e os restantes 3 pacientes (casos 2,10 e 13) estavam bem 1 ano após o ato cirúrgico.

Em um paciente (caso 11) encontramos aracnoidite crônica inespecifica envolvendo o nervo facial e o resultado terapêutico foi bom.

Em 7 pacientes (casos 3, 5, 6, 7, 8, 9 e 12) encontramos uma alça da artéria cerebelar anterior e inferior em intimo contato com o nervo facial (Fig. 1). Um destes pacientes (caso 6) apresentava também malformação de Arnold-Chiari. Em 5 pacientes deste grupo o espasmo foi totalmente abolido; em um (caso 5) o espasmo permaneceu inalterado. Um paciente (caso 9) faleceu em consequiencia de hematoma extra-dural por queda e traumatismo craniano no $3^{\circ}$ dia pós-operatório; desde 0 ato cirúrgico não apresentava espasmo hemifacial.

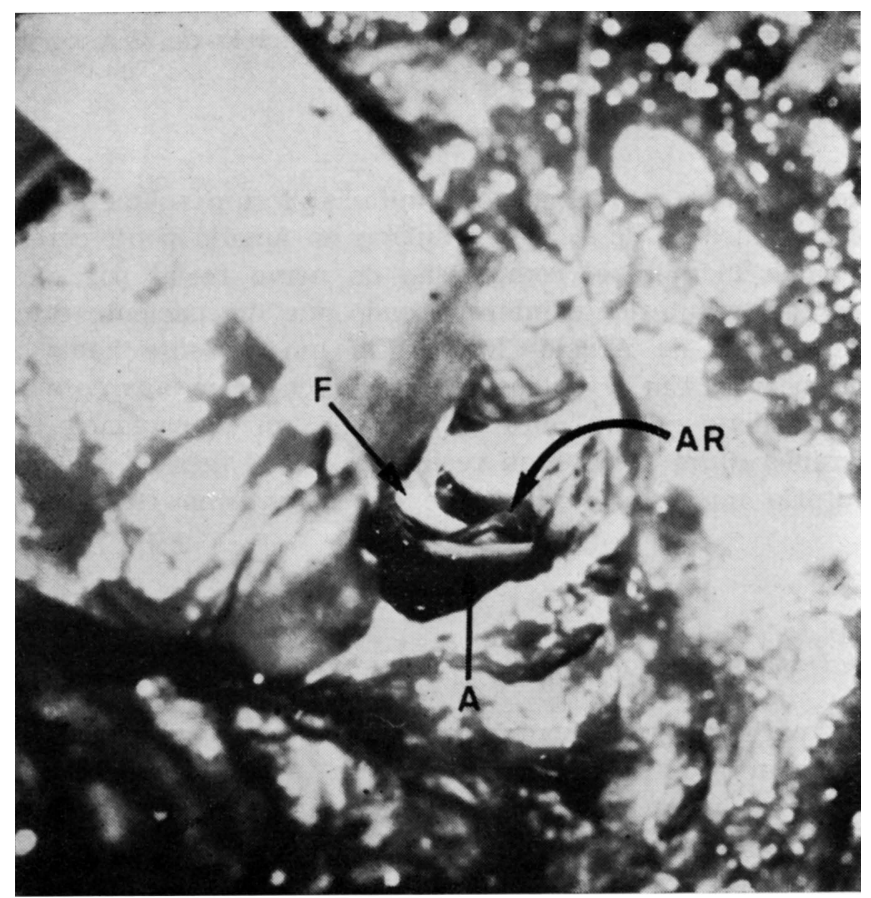

Fig. 1 - Caso 9. Exposição cirúrgica do ângulo ponto-cerebelar, vendo-se alça da artéria cerebelar anterior $e$ inferior ( $A R$ ) interposta entre os nervos auditivo (A) e facial (F).

\section{COMENTARIOS}

A neurolise do facial consiste em discreto massageamento do nervo no ângulo ponto-cerebelar a fim de desfazer o intimo contato entre os axônios sensitivos e motores ao nivel da bainha isolante lesada que permite a passa- 
gem de estímulos trans-axonais. Parece-nos que a intensidade desta massagem é ideal quando produzir discretíssima paresia facial no pós-operatório imediato, a qual desaparecerá em poucas semanas. Se o massageamento for insuficiente não produzirá paresia facial, porém corre-se o risco de não abolir o espasmo. Acreditamos que isso tenha acontecido na paciente S.M.R. (caso 1). Ao contrário, sendo muito intensa poderá ocasionar paralisia ou paresia acentuada: isso ocorreu em 2 pacientes (casos 6 e 13), porém, com recuperação espontânea quase total; em um destes pacientes (caso 13) ocorreu também hipoacusia.

Havendo malformação vascular em contato com o nervo, este deve ser isolado com um fragmento de Gelfoam.

Nos casos inalterados após ato cirúrgico corretamente conduzido e, principalmente, naqueles em que não é encontrada compressão do nervo, deve-se ter em mente a possibilidade da lesão estar situada no segmento intratemporal do facial. Então, a secção do intermediário de Wrisberg ${ }^{6}$ aliviaria o espasmo.

\section{R E S U M O}

Quatorze pacientes com espasmo hemifacial foram submetidos a exploração cirúrgica e neurolise do $7 .^{\circ}$ par craniano no ângulo ponto-cerebelar. Em 7 pacientes havia indubitável compressão do nervo facial por alça anômala da artéria cerebelar anterior e inferior sendo que um paciente também apresentava malformação de Arnold-Chiari. Em um paciente havia aracnoidite envolvendo o nervo. Em 6 outros, o nervo achava-se aparentemente livre. Houve alivio imediato e duradouro do espasmo em 10 pacientes, 2 permaneceram inalterados e um apresentou recidiva após 10 meses. Houve um óbito no pós-operatório imediato, conseqüente a traumatismo craniano por queda do paciente.

\section{S U M M A R Y}

\section{Hemifacial spasm: report of 14 cases submitted to surgical treatment}

Fourteen patients with hemifacial spasm were submitted to surgical exposure and neurolysis of the seventh nerve at the cerebellopontine angle. Seven patients had an anomalous anterior inferior cerebellar artery pulsating on the nerve, being present in one patient also Arnold-Chiari malformation. Another patient had chronic arachnoiditis involving the facial nerve. In 6 patients no abnormalities were found in the posterior fossa. There was complete relief of the spasm in 10 patients, 2 were unchanged, 1 had recurrence 10 months later and 1 died in the post-operative period due to head injury sustained during a fall.

\section{R E FER E N C I A S}

1. CAMBELl, E. \& KEEDY, C. - Hemifacial spasm: a note on the etiology in two cases. J. Neurosurg. 4:342, 1947.

2. CUSHING, H. - Tumors of the Nervus Acusticus and the Syndrome of the Cerebellopontile Angle. Hafner Publishing Co., New York-London, 1963. 
3. ECKMAN, P.; KRAMER, R. A. \& ALTROCCHI, P. H. - Hemifacial spasm. Arch. Neurol. (Chicago) 25:81, 1971.

4. EHNI, G. \& WOLTMAN, H. W. - Hemifacial spasm. Arch. Neurol. Psychiat. (Chicago) 53:205, 1945.

5. GARDNER, W. J. - Trigeminal neuralgia. In Clinical Neurosurgery, Vol. 15, Edited by Ojeman, R. G.. The Williams \& Wilkins Co., Baltimore, 1968.

6. GARDNER, W. J. \& DOHN, D. F. - Trigeminal neuralgia, hemifacial spasm, Paget's disease. Significance of this association. Brain 89:555, 1966.

7. GARDNER, W. J. \& SAVA, G. A. - Hemifacial spasm - a reversible pathophysiologic state. J. Neurosurg. 19:240, 1962 .

8. GERMAN, W. J. - Surgical treatment of spasmodic facial tic. Surgery 11:912, 1942 .

9. GREENWOOD, J. - The surgical treatment of hemifacial spasm. J. Neurosurg. 3:506, 1946 .

10. HARRIS, W. \& WRIGHT, A. D. - Treatment of clonic facial spasm. Lancet $1: 657,1932$.

11. KEMPE, L. G. \& SMITH, D. R. - Trigeminal neuralgia, facial spasm, intermedius and glossopharyngeal neuralgia with persistent carotid basilar anastomosis. J. Neurosurg. 31:445, 1969.

12. KRAUSE, F. - Chirurgie du Cerveau et de la Moelle Épinière. Tome II. Société D'Éditions Scientifiques et Médicales, Paris, 1912.

13. LAINE, M. E. \& NAYRAC, M. - Hémispasme facial guéri par intervention sur la fosse postérieure. Rev. Neurol. (Paris) 80:38, 1948.

14. MAGUN, R. \& ESSLEN, E. - Electromyographic study of reinnervated muscle and of hemifacial spasm. Amer. J. Phys. Med. 38:79, 1959.

15. NOSIK, W. A. \& WEIL, A. A. - Selective partial neurectomy in hemifacial spasm and the electrophysiologic selection of patients. J. Neurosurg, 13:596, 1956.

16. POPPEN, J. L. - An Atlas of Neurosurgical Techniques. W. B. Saunders Co., Philadelphia-London, 1960.

17. SCOVILLE, W. B. - Partial section of proximal seventh nerve trunk for facial spasm. Surg. Gynec. Obstet. 101:495, 1955.

18. TEASDAL, R. D. \& SALMAN, S. D. - Mass facial movements: electromyographic evidence for misdirection. Neurology (Minneapolis) 21:652, 1971.

19. WARTENBERG, R. - Hemifacial spasm. A clinical and pathophysiological study. Oxford University Press, New York, 1952.

20. WILSON, S. A. K. - Edited by Bruce, A. N. - Neurology, Volume 3. Butterworth \& Co. Ltd., London, 1955.

Disciplina de Neurocirurgia — Faculdade de Ciências Médicas da Santa Casa Rua Cesário Motta Junior 112 - 01221 São Paulo, SP - Brasil. 